Service social

\title{
Évaluation d'un programme d'implication parentale lors du placement du jeune en centre de réadaptation
}

\section{Gilles Mireault, André Beaudoin, Gaétan Paquet et Élise Champagne}

Volume 47, numéro 3-4, 1998

Enfance : enjeux et réalités 2000

URI : https://id.erudit.org/iderudit/706798ar

DOI : https://doi.org/10.7202/706798ar

Aller au sommaire du numéro

Éditeur(s)

École de service social de l'Université Laval

ISSN

1708-1734 (numérique)

Découvrir la revue

Citer cet article

Mireault, G., Beaudoin, A., Paquet, G. \& Champagne, É. (1998). Évaluation d'un programme d'implication parentale lors du placement du jeune en centre de réadaptation. Service social, 47(3-4), 137-167. https://doi.org/10.7202/706798ar
Résumé de l'article

Le présent article rend compte de l'évaluation d'un programme de réadaptation pour les jeunes placés au Centre jeunesse de Québec. Le programme " Transfert des acquis », instauré en 1996, propose de mobiliser, dès le début du placement, le jeune et ses parents dans une démarche d'apprentissage axée sur la résolution des problèmes vécus par la famille et sur le retour éventuel du jeune au sein de cette dernière. Les résultats de l'évaluation montrent que le principe d'impliquer les parents dans la démarche de réadaptation entraîne des changements positifs dans la perception du placement chez le jeune et chez les parents et concourt à augmenter les séjours du jeune dans sa famille au cours du placement. Le programme démontre qu'il est possible, avec le soutien approprié, de mobiliser les parents et le jeune et d'accélérer ainsi le retour de ce dernier dans la famille. 


\section{Évaluation d'un programme d'implication parentale lors du placement du jeune en centre de réadaptation}

Gilles MIREAULT

André BEAUDOIN

Gaétan PAQUET

Élise CHAMPAGNE

Le présent article rend compte de l'évaluation d'un programme de réadaptation pour les jeunes placés au Centre jeunesse de Québec. Le programme "Transfert des acquis », instauré en 1996, propose de mobiliser, dès le début du placement, le jeune et ses parents dans une démarche d'apprentissage axée sur la résolution des problèmes vécus par la famille et sur le retour éventuel du jeune au sein de cette dernière. Les résultats de l'évaluation montrent que le principe d'impliquer les parents dans la démarche de réadaptation entraîne des changements positifs dans la perception du placement chez le jeune et chez les parents et concourt à augmenter les séjours du jeune dans sa famille au cours du placement. Le programme démontre qu'il est possible, avec le soutien approprié, de mobiliser les parents et le jeune et d'accélérer ainsi le retour de ce dernier dans la famille.

In 1996, le Centre jeunesse de Québec initiated a rehabilitation program for adolescents in residential care. The initiative proposes to involve, at the onset of the program, both parents and their adolescent in dealing with their problems and planning for a home return. The evaluation results suggest that parental involvement can change both the parents and the adolescent's perception about the residential care stay. Moreover, home visits during the residential care period are increased and chances for returning home at the end of the program are better. The program demonstrates that for these families, working intensively towards a return of their youngster is possible and feasible with the appropriate support. 
La place accordée aux parents lors du placement de leur jeune en centre de réadaptation est de plus en plus l'objet d'attention dans le cadre du plan d'intervention offert aux familles. La nécessité d'impliquer les parents dans les différentes mesures concernant le bien-être des enfants, aussi bien dans leur famille que lors d'un placement, est de plus en plus reconnue.

Le présent article rend compte des résultats de l'évaluation du programme "Transfert des acquis » (TDA) expérimenté au Centre jeunesse de Québec entre janvier 1996 et décembre 1997 auprès de 40 jeunes de 14 à 17 ans placés en centre de réadaptation et de leur famille. Dans son ensemble, ce programme vise à impliquer les parents et les jeunes pendant leur placement en centre de réadaptation en insistant sur la résolution des conflits, l'acquisition de nouvelles habiletés de communication et de nouvelles façons de faire dans leurs interactions. II sera question de la problématique à l'origine de la présente étude, de la nature et des éléments constitutifs du programme "Transfert des acquis ", de la méthode utilisée pour l'évaluation, des caractéristiques des jeunes et des parents concernés et des principaux résultats constatés en ce qui regarde les effets du programme.

\section{LA PROBLÉMATIQUE DE LA PLACE DES PARENTS DANS LE PLACEMENT DES ADOLESCENTS}

Sans dénier la nécessité du placement dans les situations où le bien-être de l'enfant est compromis et lorsque cela est nécessaire pour sa réadaptation, cette mesure doit s'inscrire dans une perspective de plan d'intervention qui implique les parents et qui tient compte du développement de l'enfant à long terme (Blythe et collab., 1994; Conseil des affaires sociales, 1990; Roberge, 1995).

Cette orientation est appuyée par la documentation scientifique sur l'implication des parents lors du placement du jeune. Bien que la plupart des écrits consultés provenant de la littérature publiée aux États-Unis ne définissent pas clairement ce qu'il faut entendre par implication parentale (family involvement, parental involvement), plusieurs recherches démontrent que les programmes de réadaptation, qui permettent le maintien des liens entre le parent et l'enfant pendant le placement, obtiennent des résultats qui se maintiennent plus longtemps dans le milieu naturel, après le 
retour du jeune dans sa famille (Allerhand et collab., 1966; Taylor et Alpert, 1973; Whittaker et Garbarino, 1983). La documentation scientifique suggère également que ces programmes permettent aussi le développement d'habiletés parentales qui se maintiennent après le placement (Berkowitz et Graziano, 1972; Johnson et Katz, 1973). Ainsi, plusieurs auteurs considèrent que l'implication parentale et le soutien de la famille, par le biais d'une interaction continue entre les parents, les jeunes et les intervenants pendant le processus de réadaptation du jeune, sont l'un des éléments prédictifs les plus puissants de la capacité du jeune à réussir à s'adapter dans son milieu naturel et à transférer les gains thérapeutiques après son placement (Krona, 1980; Whittaker, 1981; Fanshel, 1975; Rowe, Cain, Hundleby et Keane, 1984; Taylor et Alpert, 1973; Whittaker et Maluccio, 1986; Whittaker et Garbarino, 1983).

La documentation scientifique québécoise comporte peu de programmes spécifiquement décrits comme visant une plus grande implication parentale dans un contexte de placement. De fait, nous n'avons recensé aucun programme évalué ou décrit sur le plan de son impact. Les auteurs consultés (Simard, Vachon et Moisan, 1991; Leblanc, 1995; Pauzé, Béchard et Toupin, 1993) semblent plutôt s'attarder à la famille d'un point de vue plus descriptif en mesurant le fonctionnement familial.

\section{LE PROGRAMME « TRANSFERT DES ACQUIS » ET SON CONTEXTE D'APPLICATION}

Le programme "Transfert des acquis » (Paquet, 1995) est né de la préoccupation de rendre les meilleurs services possible aux jeunes qui sont confiés au Centre jeunesse pour placement. À cet égard, plusieurs jeunes et leur famille, malgré les difficultés auxquelles ils doivent faire face, conservent la volonté de continuer à vivre ensemble dans un futur rapproché. Malheureusement, les circonstances présentes au moment du placement sont telles que cette volonté est mise en doute par les principaux protagonistes. Dans cette perspective, le programme «Transfert des acquis " propose une approche centrée sur l'implication du jeune et de ses parents dans le processus de résolution des conflits qui les opposent, et ce, dès les premiers jours du placement.

Par l'acquisition de nouvelles habiletés de communication et par 
l'expérimentation de nouvelles façons de faire dans le milieu de vie naturelle du jeune, le "Transfert des acquis " renforce les partenaires familiaux dans leur volonté de vivre à nouveau ensemble dans un avenir rapproché.

La caractéristique essentielle de la clientèle visée par le programme est l'ouverture démontrée par le jeune et ses parents à revivre ensemble et à collaborer dans la recherche de moyens afin de régler la situation conflictuelle. Le milieu naturel du jeune (famille, école) demeure son principal milieu de vie. La famille a le soin de gérer elle-même ce qui va bien et de maintenir les activités dans le milieu qui sont positives. Concrètement, des séjours intensifs de courte et de moyenne durée en centre de réadaptation permettent à l'adolescent d'acquérir des compétences personnelles et sociales par des enseignements individuels ou en groupe. Parallèlement, le parent s'engage dans un programme d'acquisition ou de développement de compétences parentales directement en lien avec la programmation spécifique de son fils. L'objectif global du projet demeure de maintenir le "lit chaud » dans la famille et le "bureau chaud » à l'école d'origine, pour en venir ultérieurement à permettre aux usagers de devenir indépendants des services de réadaptation. Un modèle théorique emprunté à la psychologie cognitive (Tardif, 1992) est à la base d'un ensemble de pistes d'intervention qui visent à favoriser et à mesurer l'engagement, la participation et la persistance des usagers durant le projet depuis l'accueil jusqu'à la fin du placement.

À partir de six critères d'admission, les jeunes sont rapidement dirigés vers le programme pour une évaluation plus poussée de la motivation et des capacités de la famille à évoluer dans le cadre du programme. L'accent est mis autant sur les secteurs actualisants (ce qui va bien) que sur la problématique (ce qui va moins bien). L'objectif fondamental demeure que le jeune puisse poursuivre ses activités positives dans son milieu et qu'il ne vienne au centre que pour "travailler" les zones problématiques. Nous pensons, par exemple, au maintien du rôle social (travail, école), à la fréquentation de pairs positifs, à l'affiliation à un groupe organisé (scouts, cadets, etc.), aux sorties dans les maisons de jeunes ou à l'inscription à une activité de loisirs (hockey, basket-ball, etc.). Cet inventaire doit se faire rapidement, dans les deux ou trois semaines suivant l'inscription de la famille dans le projet. 
Les sessions d'apprentissage se font en groupe, et pour les adolescents et pour les parents. Les mêmes thèmes sont abordés dans chaque groupe, dans le même ordre et suivant des échéanciers similaires. Une session s'étend sur 7 semaines, soit 7 rencontres hebdomadaires de 2 heures. Le processus prévoit des enseignements parallèles (jeunes et parents séparés) entrecoupés d'échanges en plénière (jeunes et parents regroupés) où les participants partagent leurs points de vue sur les contenus abordés au cours des semaines précédant la jonction. Les familles ont accès à trois différents blocs d'apprentissage: Vivre ensemble (communication) - Grandir ensemble (toxicomanie) S'asseoir ensemble (gestion des émotions). Les éducateurs et travailleurs sociaux des unités de vie concernées se chargent de l'animation et de la coanimation des activités.

Enfin, la réorganisation du centre de réadaptation est un préalable nécessaire à la bonne marche du projet, en particulier dans les milieux où les référents institutionnels caractérisent le quotidien des intervenants. Le principal défi réside dans la nécessité d'un changement de pratique à l'interne et d'une ouverture vers l'extérieur en fonction des exigences du projet. II faut spécifier à cet effet la nécessité pour l'internat de passer d'une programmation de groupe à une programmation davantage individualisée. Rappelons que la réorganisation du milieu s'effectue en même temps que l'implantation du projet, avec toutes les difficultés que cette façon de faire implique: clientèle mixte, programmation mixte, ajout et modification de certaines tâches, aménagement différent des espaces physiques, formation du personnel. Les structures du centre de réadaptation ont également à s'ajuster en fonction des besoins des jeunes et de leur famille. C'est dire, par exemple, que l'horaire de travail des intervenants répond aux disponibilités réelles des familles qu'ils accompagnent de façon plus intensive, que l'agenda des activités d'apprentissage et des activités cliniques tient compte du calendrier des secteurs actualisants (travail - école - loisir) des adolescents. À cet effet, les éducateurs de l'internat effectuent plus de présence en milieu familial. On parle ici d'environ un soir de présence supplémentaire par semaine et d'une concentration de l'effectif en fonction de la distribution des activités dans le calendrier hebdomadaire de la programmation. Le centre doit également ouvrir grandes ses portes aux familles et assouplir ses règles liées au temps de visite. Par 
exemple, les parents sont invités à prendre des repas, à se présenter aux unités de façon ponctuelle et à participer à des activités planifiées au centre de réadaptation. La collaboration des services techniques et administratifs est nécessaire à cette démarche. De nouvelles activités se greffent aux activités cliniques traditionnelles. Des repas communautaires, des activités sociales, des soirées de formation et d'information sont offertes régulièrement aux familles. Des ententes précises avec les services alimentaires et le service des activités, par exemple, sont nécessaires au bon fonctionnement de plusieurs de ces activités.

\section{LA MÉTHODE D'ÉVALUATION}

L'évaluation a consisté en trois opérations distinctes d'évaluation : l'étude d'implantation (Beaudoin et Aucoin, 1996), une analyse quantitative des effets et une analyse qualitative du programme (Mireault, Beaudoin, Paquet et Champagne, 1998).

L'étude d'implantation a été effectuée à deux moments, au printemps et à l'automne $1996^{1}$, par une collecte de données sur les participants et les actions des intervenants dans le cadre du programme. Le devis retenu pour la mesure quantitative des effets est une comparaison de deux groupes de jeunes ayant séjourné au Centre jeunesse de Québec dans le cadre d'un placement. Le premier groupe sélectionné est celui des jeunes qui ont participé au programme "Transfert des acquis » (groupe TDA) à partir du $1^{\mathrm{er}}$ janvier 1996. Quarante jeunes inscrits dans le programme à partir du $1^{\mathrm{er}}$ janvier 1996 et dont le placement s'est terminé au plus tard le 30 juin 1997 ont été retenus. Le second groupe (groupe TÉMOIN) $(n=77)$ est constitué de jeunes dont le placement s'est terminé avant le 31 décembre 1995, soit juste avant le début des activités du programme. La comparaison des deux groupes sur les mesures d'effets se fait après-coup (ex-post). Sur le plan méthodologique, la comparaison de deux groupes sans

\footnotetext{
L'évaluation du programme «Transfert des acquis » faisait partie des conditions pour l'obtention de la subvention de 239680 \$ du Fonds de soutien à l'innovation du ministère de la Santé et des Services sociaux destinée à appuyer l'expérimentation du programme "Transfert des acquis ». Une contribution de $23900 \$(10 \%)$ a été séparée du budget de fonctionnement pour soutenir l'évaluation.
} 
mesures préalables permet difficilement de déterminer l'équivalence des deux groupes au départ. Pour atténuer l'impact de cette contrainte, nous avons sélectionné les sujets des deux groupes sur des variables qui peuvent influencer leurs résultats au programme. Ainsi, nous avons tenu compte de la loi en vertu de laquelle les jeunes ont été placés (les cas de Loi sur les jeunes contrevenants ont été exclus) et de l'unité de vie lors du placement. Cette stratégie augmente les chances d'obtenir une meilleure équivalence des groupes sans pour autant la garantir. Par ailleurs, des données sur le milieu de vie avant le placement, sur le temps de placement au cours des deux dernières années qui ont précédé l'épisode de placement étudié, sur la structure familiale et d'autres ont été colligées pour servir de base de comparaison entre les deux groupes et vérifier leur impact sur les variables dépendantes.

Du coté des mesures d'effets (variables dépendantes), quatre mesures ont été retenues:

- Le pourcentage de temps passé à l'extérieur du centre de réadaptation lors du placement (défini par les couchers en milieu familial).

- Le milieu de vie après le placement (retour en famille ou continuité en centre d'accueil).

- Le nombre de jours placés 3 mois après la fin du placement étudié.

- Le nombre de jours placés entre 3 et 6 mois après la fin du placement étudié.

Des analyses de régression permettront de rendre compte de l'apport spécifique de la variable groupe (groupe TDA) tout en isolant l'apport des autres variables. Ainsi, le poids de chaque variable sur les mesures d'effets est déterminé et quantifié statistiquement.

La troisième opération a consisté en une analyse qualitative effectuée à partir d'entrevues de personnes clés engagées dans le programme à différents égards. Huit paires parents-jeunes ainsi que deux parents et deux jeunes non appariés ont participé aux 
entrevues. Pour la sélection des jeunes et des parents, le temps écoulé depuis la fin du programme (plus ou moins de 6 mois) ainsi que l'appréciation clinique des résultats (retour ou non à la maison, participation, observation clinique) ont servi de critères de sélection des participants. Les entrevues ont porté sur différents thèmes concernant les difficultés du jeune, les moyens utilisés pour résoudre celles-ci, l'expérience du programme TDA et les effets du programme.

Les personnes suivantes impliquées dans le programme ont aussi été rencontrées en entrevue: sept éducateurs dans les unités concernées, deux conseillers cliniques, deux chefs d'unité, deux travailleurs sociaux, l'éducateur responsable de l'implantation et du déroulement du programme et un des principaux concepteurs du "Transfert des acquis". Dans ces entrevues, en plus de l'information sur leur formation et sur leur expérience de travail, les thèmes abordés visaient à connaître leur opinion concernant le fonctionnement du programme, ses facteurs de réussite et d'échec, ses résultats ou effets, son avenir et leurs suggestions si c'était à refaire.

Le contenu de chacune des entrevues a d'abord fait l'objet d'un résumé le plus tôt possible après l'entrevue. Par la suite, une analyse thématique transversale des contenus recueillis auprès des jeunes, parents, éducateurs et autres intervenants a été effectuée afin de dégager les points communs et les divergences.

\section{LES ADOLESCENTS ET LES PARENTS PARTICIPANT AU TDA}

\section{Les adolescents}

Au total, 54 jeunes et leurs familles ont participé au projet "Transfert des acquis » durant les deux années d'expérimentation. II y a eu une moyenne de 14 jeunes participant mensuellement au programme. Les données qui suivent sont tirées des 40 dyades parents-jeunes qui ont fait l'objet de l'évaluation du programme. Dix-sept jeunes $(42,5 \%)$ ont 16 ans, vingt $(50 \%)$ sont âgés de 15 ans et un de 17 ans, pour une moyenne de 15,9 ans. Quatorze jeunes (35\%) proviennent de familles biparentales composées des deux parents naturels du jeune, alors 
que vingt-cinq $(62,5 \%)$ vivent avec leur mère $(52,5 \%)$ ou leur père $(10 \%)^{2}$.

Soixante-quinze pour cent (30 adolescents) des participants au "Transfert des acquis " sont de niveau $3^{\mathrm{e}}$ secondaire ou moins. Onze jeunes $(27,5 \%)$ ont un cheminement scolaire particulier. La plupart des jeunes $(80 \%)$ ont un retard scolaire. Ils ont presque tous vécu des difficultés scolaires à un moment ou à un autre. Ces difficultés deviennent plus accentuées pendant le secondaire et pour la plupart d'entre eux ils continuent à vivre des difficultés scolaires.

En ce qui concerne l'historique des services reçus par ces jeunes au cours de l'enfance et de l'adolescence, pour seize cas (40\%) il y a mention de suivi familial pendant l'adolescence avant le " Transfert des acquis » et dans dix cas (25\%) de suivi en centre local de services communautaires. Les services d'évaluation et de suivi psychologique avaient déjà été utilisés pendant l'enfance pour un certain nombre de jeunes $(20 \%)$, mais on y a recouru proportionnellement un peu plus pendant l'adolescence $(25 \%)$. Six jeunes $(15 \%)$ ont été placés en famille d'accueil durant l'enfance, un seul en foyer de groupe et cinq (12,5\%) en centre de réadaptation. À l'adolescence, trente jeunes, soit les trois quarts de la population du "Transfert des acquis ", ont déjà fait l'objet d'un placement en institution antérieur au placement étudié, douze $(30 \%)$ ont été placés dans un foyer de groupe et sept $(12,5 \%)$ en famille d'accueil. Les services spécialisés en toxicomanie, qui n'ont fait l'objet d'aucune utilisation durant l'enfance, ont été utilisés six fois (15\%) à l'adolescence.

Les problèmes mis en évidence chez les jeunes du «Transfert des acquis " sont les difficultés comportementales. Celles-ci sont mentionnées pour trente-huit (95\%) des quarante jeunes du programme. On est dans la très grande majorité des cas $(80 \%)$ en présence de plus d'un problème de comportement et dans huit cas $(20 \%)$ on est en présence de cinq mentions de ce type de difficultés. Les problèmes de comportement prennent surtout les formes suivantes : utilisation d'alcool ou drogue (65\% des cas),

2 Onze des adolescents vivent avec leur mère ou à l'intérieur de familles recomposées. 
fugue $(47,5 \%)$, violence $(42,5 \%)$, absentéisme scolaire (45\%) et conflits avec les pairs et les adultes (42,5\%); il y a aussi à un moindre degré des problèmes de retrait ou de passivité $(25 \%)$ ou d'hyperactivité (12,5\%).

Même s'il ne s'agit pas de jeunes pris en charge selon la Loi sur les jeunes contrevenants, la présence d'agirs délinquants est mentionnée pour vingt-cinq jeunes $(62,5 \%)$ et il y a présence de trois agirs délinquants et plus chez cinq d'entre eux. Ces agirs prennent la forme de délits contre la propriété (45\%), d'infractions (35\%), de délits contre la personne $(27,5 \%)$ ou autres $(15 \%)$. Viennent ensuite des problèmes liés à la performance scolaire ou des problèmes sur le plan intellectuel, qui sont présents chez dixneuf jeunes $(47,5 \%)$. II s'agit de problèmes de retard scolaire (30 \%) et de problèmes d'apprentissage $(7,5 \%)$ ou des deux à la fois (10\%). La maltraitance est présente chez huit jeunes $(20 \%)$. Ses manifestations principales sont réparties également sous les formes d'abus physique, d'abus sexuel ou de négligence.

Pour sept jeunes $(17,5 \%)$, il y a mention de problèmes de santé mentale qui prennent la forme d'états dépressifs (10\%), d'idéations suicidaires $(7,5 \%)$; on note une mention pour chacun d'automutilation ou de phobie et une autre mention non précisée. II y a très peu de problèmes de santé physique constatés : seul un handicap visuel et auditif est signalé.

\section{Les parents}

La moyenne d'âge des pères $(n=19)$ est de 46 ans, avec un écart-type de 5,3 , alors que pour les mères $(n=25)$ la moyenne est de 43 ans (écart-type de 8 ). II y a trois mères (12\%) dans les catégories de 35 ans et moins, alors que les plus jeunes pères se situent dans la catégorie des 35-39 ans, où ils sont encore moins nombreux proportionnellement que les mères. Pour ce qui est des autres catégories d'âge, la répartition est assez semblable.

La principale source de revenus aussi bien des pères que des mères est l'emploi. D'ailleurs, il n'y a à peu près pas de différence entre les pères et les mères quant aux sources de revenus. On ne note pas de différence dans les revenus des parents du «Transfert des acquis » à partir des données fournies par seize 
pères et seize mères, si l'on fait exception d'un nombre un peu plus élevé de pères qui ont des revenus de $50000 \$$ et plus.

Pour ce qui est de la scolarité, celle des mères paraît un peu plus élevée que celle des pères: ainsi, $47,6 \%$ des mères ont une scolarité de niveau collégial et universitaire, alors que cette proportion n'est que de $25 \%$ chez les pères.

Les difficultés recensées par les intervenants en entrevue avec les parents représentent une gamme assez étendue de problèmes familiaux. Autant chez les pères que chez les mères, on constate la présence de trois problèmes relationnels: relations parents-enfants, manque de disponibilité parentale et problèmes liés à l'encadrement et à l'exercice de la discipline. Ce sont les problèmes les plus souvent mentionnés. Certains problèmes, comme le divorce, la séparation, le décès du conjoint, la perte d'emploi ou le changement d'emploi, sont aussi signalés, aussi bien chez les pères que chez les mères. Par ailleurs, les problèmes de structure familiale (séparation, recomposition) seraient deux fois plus présents chez les mères (52 \%) que chez les pères $(26,3 \%)$, alors que les problèmes de discorde conjugale seraient plus présents chez les pères que chez les mères.

\section{LES ACTIVITÉS DU PROGRAMME}

La présente section trace un aperçu des activités effectuées concernant les parents et les jeunes dans le cadre du programme à partir des données de l'étude d'implantation (Beaudoin et Aucoin, 1996). Les activités mentionnées ici sont celles des éducateurs du "Transfert des acquis ». Elles ont été compilées pour 30 jeunes sur un minimum de 6 semaines ( 30 jours) jusqu'à un maximum de 12 semaines (60 jours). Les activités rapportées couvrent 4,8 périodes (soit 9,6 semaines) en moyenne pour chacun des 30 jeunes concernés. Le nombre total d'activités recensées est de 5384 activités pour l'ensemble de la période de la collecte des données sur l'exposition au programme, soit une moyenne de 183,2 activités par période de dix jours ou de deux semaines utilisée ici comme période de référence. 
Quant à leur contenu, les activités se regroupent comme suit :

\begin{tabular}{|l|c|c|}
\hline \multicolumn{1}{|c|}{ Contenu des activités } & $\begin{array}{c}\text { Nombre } \\
\text { moyen par } \\
\text { jeune pour } \\
\text { 10 jours }\end{array}$ & $\begin{array}{c}\text { Pourcentage } \\
\text { de l'ensemble des } \\
\text { activités }\end{array}$ \\
\hline $\begin{array}{l}\text { Activités d'apprentissage exécutées } \\
\text { en lien avec les ateliers parents- } \\
\text { adolescents }\end{array}$ & 1,9 & $31,3 \%$ \\
\hline $\begin{array}{l}\text { Activités régulières pour appuyer les } \\
\text { contacts et l'interaction entre les } \\
\text { jeunes et leurs parents }\end{array}$ & 1,9 & $30,6 \%$ \\
\hline $\begin{array}{l}\text { Activités d'intervention clinique } \\
\text { exécutées dans le cadre du } \\
\text { programme « Transfert des acquis » }\end{array}$ & 1,4 & $23,6 \%$ \\
\hline $\begin{array}{l}\text { Autres activités du programme } \\
\text { incluant les activités d'évaluation }\end{array}$ & 0,9 & $13,7 \%$ \\
\hline
\end{tabular}

La répartition des activités selon les objectifs poursuivis est la suivante :

\begin{tabular}{|l|c|c|}
\hline \multicolumn{1}{|c|}{ Objectifs des activités } & $\begin{array}{c}\text { Nombre } \\
\text { moyen par } \\
\text { jeune pour } \\
\mathbf{1 0} \text { jours }\end{array}$ & $\begin{array}{c}\text { Pourcentage de } \\
\text { l'ensemble des } \\
\text { activités }\end{array}$ \\
\hline $\begin{array}{l}\text { Favoriser les activités communes } \\
\text { jeunes et famille }\end{array}$ & 1,4 & $24,0 \%$ \\
\hline $\begin{array}{l}\text { Expérimenter des modes de } \\
\text { communication et d'interaction } \\
\text { parents-jeunes }\end{array}$ & 1,1 & $17,6 \%$ \\
\hline $\begin{array}{l}\text { Fournir du soutien et de } \\
\text { l'encouragement pour } \\
\text { l'accomplissement des exigences } \\
\text { du programme }\end{array}$ & 0,8 & $14,1 \%$ \\
\hline $\begin{array}{l}\text { Retour avec le jeune sur les différentes } \\
\text { activités accomplies avec les parents }\end{array}$ & 0,7 & $12,3 \%$ \\
\hline $\begin{array}{l}\text { Objectifs de clarification, de } \\
\text { préparation des rencontres et ateliers, } \\
\text { de réalisation des ateliers } \\
\text { et de concertation }\end{array}$ & 1,3 & $22,2 \%$ \\
\hline Autres types d'objectifs & 0,6 & $9,8 \%$ \\
\hline
\end{tabular}


La plus grande partie des activités propres au programme se déroulent au centre de réadaptation. Pour chaque période de 10 jours, il y a en moyenne 84,5 activités accomplies en centre de réadaptation, soit 2,8 par jeune; ces activités représentent $57,4 \%$ de l'ensemble des activités du programme. Les 44 activités en moyenne (1,5 pour chaque jeune) qui se déroulent dans la famille pour une période de 10 jours représentent quant à elles près de $30 \%$ de l'ensemble des activités. Par ailleurs, seulement 3,2\% des activités se déroulent à l'école.

En ce qui concerne la durée, les activités qui se réalisent dans la famille sont de loin celles qui ont la durée la plus longue, soit une moyenne de 20 h 47 par jeune pour chaque période de 10 jours. Comme il fallait s'y attendre, comparativement à la durée passée dans la famille, la durée des activités en centre de réadaptation est beaucoup plus courte. Elle ne représente que 2 h 35 en moyenne pour chaque jeune par période de 10 jours, c'est-à-dire environ une durée huit fois moins grande.

\section{LES EFFETS DU PROGRAMME}

Cette section présente les résultats des analyses quantitatives faites sur les mesures d'effets. En premier lieu une comparaison des groupes TDA et TÉMOIN, portant sur les résultats bruts, est présentée. Les principaux résultats des analyses permettent ensuite de mieux saisir quelles variables peuvent influencer les différences brutes enregistrées entre les deux groupes. En troisième lieu, les résultats des analyses de régression serviront à mettre en valeur les apports spécifiques de certaines variables sur les résultats, en particulier la variable groupe, qui représente l'exposition au programme TDA.

\section{Comparaisons brutes des groupes TDA et TÉMOIN quant aux mesures d'effets}

La présente section donne les résultats des analyses quantitatives effectuées pour mettre en valeur les effets du programme à partir des quatre mesures d'effet retenues, soit le pourcentage de temps passé dans la famille (couchers), le milieu de vie à la fin du placement, le nombre de jours placés 3 mois et 6 mois après la fin du placement. Le tableau 1 présente une comparaison brute des 
groupes TDA et TÉMOIN sur les mesures d'effets retenues pour l'évaluation. Le pourcentage de temps passé en dehors du centre de réadaptation diffère de manière statistiquement significative entre les groupes TDA et TÉMOIN, et ce, à l'avantage du groupe TDA $(43,2 \%$ contre $28,9 \%)$. En ce qui concerne le retour en milieu familial, aucune différence significative n'est notable entre les deux groupes. On ne note également aucune différence significative quant au nombre de jours placés 3 mois après le placement. Enfin, le nombre de jours placés entre 3 et 6 mois après la fin du placement est très près du seuil statistiquement significatif en faveur du groupe TDA.

Tableau 1

Résultats des tests de différence des moyennes et de variance sur les mesures d'effets pour les groupes « Transfert des acquis » et TÉMOIN

\begin{tabular}{|c|c|c|c|c|c|c|c|c|c|c|c|c|}
\hline \multirow[t]{2}{*}{ Groupes } & \multicolumn{3}{|c|}{$\begin{array}{c}\text { Pourcentage } \\
\text { moyen de temps } \\
\text { passé hors du } \\
\text { CR }\end{array}$} & \multicolumn{3}{|c|}{$\begin{array}{l}\text { Milieu de vie } \\
\text { après le } \\
\text { placement } \\
\text { étudié (famille) }\end{array}$} & \multicolumn{3}{|c|}{$\begin{array}{c}\text { Nombre moyen } \\
\text { de jours placés } \\
\text { après } 3 \text { mois }\end{array}$} & \multicolumn{3}{|c|}{$\begin{array}{l}\text { Nombre moyen } \\
\text { de jours placés } \\
\text { entre } 3 \text { et } 6 \text { mois }\end{array}$} \\
\hline & $\%$ & (n) & $p$ & $\%$ & (n) & $p$ & $x$ & (n) & $P$ & $x$ & (n) & $P$ \\
\hline $\begin{array}{l}\text { Transfert des } \\
\text { acquis }(n=40)\end{array}$ & 43,2 & (40) & 0,000 & 65,7 & (23) & 0,212 & 21,8 & (35) & 0,501 & 18,9 & (30) & 0,066 \\
\hline Témoin $(n=77)$ & 28,9 & (77) & & 77,1 & (54) & & 27,1 & (71) & & 29,5 & (65) & \\
\hline
\end{tabular}

Parallèlement à cette augmentation significative du temps passé à l'extérieur, nous avons regardé, à titre indicatif, la répartition des séjours à l'extérieur (avec coucher) durant la semaine. Le tableau 2 montre les pourcentages de séjour avec couchers à la maison selon leur distribution au cours de la semaine. Le pattern habituel que l'on retrouve dans les centres de réadaptation correspond à celui du groupe TÉMOIN où l'on observe que les fins de semaine (vendredi, samedi) occupent un peu plus de la moitié des congés hebdomadaires $(53,7 \%)$, alors que le groupe TDA totalise $42,6 \%$ pour ces mêmes journées. Cette différence de $11,2 \%$ se distribue tout au long de la semaine. De fait, l'augmentation du temps passé en famille la semaine par les jeunes du «Transfert des acquis " est de près de $50 \%$ pour les lundi, mardi et mercredi. Ce résultat rejoint l'objectif du programme qui est de modifier le pattern habituel d'alternance semaine et fin de semaine 
entre le centre de réadaptation et la famille. Les résultats du groupe TDA vont dans le sens d'une répartition de plus en plus uniforme des congés durant la semaine.

Tableau 2

Répartition des séjours à l'extérieur (coucher) du centre de réadaptation selon la journée de la semaine

\begin{tabular}{|c|c|c|c|c|}
\hline \multirow{2}{*}{ Journée } & \multicolumn{2}{|c|}{$\begin{array}{c}\text { Transfert des acquis } \\
(\boldsymbol{n}=\mathbf{4 0})\end{array}$} & \multicolumn{2}{c|}{$\begin{array}{c}\text { Témoin } \\
(\boldsymbol{n}=\mathbf{7 7})\end{array}$} \\
\cline { 2 - 5 } & $\%$ & $\begin{array}{c}\text { Nombre de } \\
\text { jours }\end{array}$ & $\%$ & $\begin{array}{c}\text { Nombre } \\
\text { de jours }\end{array}$ \\
\hline Dimanche & 15,6 & 513 & 15,5 & 1039 \\
\hline Lundi & 11,2 & 367 & 7,6 & 510 \\
\hline Mardi & 9,5 & 313 & 6,7 & 452 \\
\hline Mercredi & 9,9 & 321 & 6,5 & 434 \\
\hline Jeudi & 10,8 & 349 & 9,7 & 649 \\
\hline Vendredi & 20,7 & 697 & 25,7 & 1721 \\
\hline Samedi & 21,9 & 738 & 28,0 & 1873 \\
\hline $\begin{array}{l}\text { Total des couchers } \\
\text { à l'extérieur }\end{array}$ & 100 & 3298 & 100 & 6678 \\
\hline
\end{tabular}

\section{Analyses bivariées}

Ce premier regard sur les mesures d'effets montre que les résultats pour le groupe TDA sont meilleurs que ceux du groupe TÉMOIN en ce qui a trait au pourcentage de temps passé en dehors du centre de réadaptation, à la répartition des jours de congé au cours de la semaine et aux jours placés entre 3 et 6 mois après le placement. Ces résultats bruts ne tiennent pas compte des différences de départ des deux groupes. Celles-ci pourraient expliquer pourquoi certains résultats bruts diffèrent entre les deux groupes. Nous avons donc comparé le groupe TDA 
avec le groupe TÉMOIN sur quelques variables afin de relever des différences entre les deux groupes. Les tableaux croisés (non présentés) ne suggèrent aucune différence statistiquement significative entre les groupes quant au lieu de séjour lors du placement étudié, les lois sous lesquelles les jeunes ont été placés ainsi que la structure familiale. De plus, aucune différence quant à l'âge au début du placement ainsi que quant au nombre de jours placés dans les deux ans précédant le placement actuel n'est notée. Par contre, le milieu de vie avant le placement étudié indique que plus de jeunes du groupe TÉMOIN (51\%) que du groupe TDA (45\%) viennent de l'internat. Cette différence qui s'approche du niveau statistique de signification habituellement retenu est suffisamment importante et significative d'un point de vue clinique pour qu'on vérifie si elle n'est pas en relation avec certaines des mesures d'effets.

Parallèlement, des analyses bivariées entre les mesures d'effets et les mesures indépendantes retenues ont été examinées pour repérer l'effet potentiel de certaines variables sur les mesures d'effets. Le tableau 3 présente ces résultats. Ainsi, le nombre de jours placés au cours des deux dernières années précédant le placement semble en lien $(p<0,05)$ avec le nombre de jours placés à 3 mois et entre 3 et 6 mois après le placement étudié. Ces données suggèrent que les résultats des groupes aux mesures d'effets peuvent être influencés par la présence de variables qui y sont étroitement liées. Les variables qui sont significatives à 0,10 pour chacune des variables dépendantes seront retenues pour les analyses de régression subséquentes. Nous avons également tenu compte des liens statistiques étroits qu'entretiennent les variables indépendantes entre elles pour les insérer dans les analyses de régression ultérieures. 
Tableau 3

Résultats des analyses bivariées entre les variables indépendantes et les mesures d'effets

\begin{tabular}{|c|c|c|c|c|c|c|c|c|c|}
\hline & \multicolumn{2}{|c|}{$\begin{array}{l}\text { \% de temps } \\
\text { hors du C.R. }\end{array}$} & \multicolumn{3}{|c|}{$\begin{array}{c}\text { Milieu de vie } \\
\text { après le placement }\end{array}$} & \multicolumn{2}{|c|}{$\begin{array}{c}\text { Nbre de jours } \\
\text { placés } 3 \text { mois } \\
\text { après le } \\
\text { placement }\end{array}$} & \multicolumn{2}{|c|}{$\begin{array}{c}\text { Nbre de jours } \\
\text { placés entre } 3 \\
\text { et } 6 \text { mois après } \\
\text { le placement }\end{array}$} \\
\hline & \begin{tabular}{|l|} 
Moy. ou \\
valeur F
\end{tabular} & $p$ & $\begin{array}{l}\text { \% fa- } \\
\text { mille }\end{array}$ & $\begin{array}{c}\% \\
\text { CJQ }\end{array}$ & $p$ & $\begin{array}{l}\text { Moy. ou } \\
\text { valeur F }\end{array}$ & $p$ & \begin{tabular}{|l|} 
Moy. ou \\
valeur F
\end{tabular} & $P$ \\
\hline $\begin{array}{c}\text { Structure familiale } \\
\text { Parents naturels } \\
\text { Mono. et autres }\end{array}$ & $\begin{array}{l}38,2 \\
32,1\end{array}$ & 0,130 & $\begin{array}{l}80,0 \\
70,6\end{array}$ & \begin{tabular}{|l|}
20,0 \\
29,4
\end{tabular} & 0,300 & $\begin{array}{l}27,6 \\
23,7\end{array}$ & 0,620 & $\begin{array}{l}14,0 \\
30,2\end{array}$ & 0,080 \\
\hline $\begin{array}{l}\text { Lois } \\
\text { LSSSS } \\
\text { LPJ } \\
\text { LPJ + LJC }\end{array}$ & $\begin{array}{l}36,5 \\
32,9 \\
34,2\end{array}$ & 0,860 & $\begin{array}{l}70,0 \\
78,8 \\
30,0\end{array}$ & $\begin{array}{l}30,0 \\
21,2 \\
70,0\end{array}$ & 0,004 & $\begin{array}{l}21,3 \\
22,3 \\
55,4\end{array}$ & 0,139 & $\begin{array}{l}55,7 \\
19,7 \\
55,7\end{array}$ & 0,003 \\
\hline $\begin{array}{l}\text { Historique de } \\
\text { placement depuis } \\
2 \text { ans (jours) }\end{array}$ & $F=1,48$ & 0,226 & 155,0 & 236 & 0,091 & $F=9,79$ & 0,002 & $F=7,73$ & 0,019 \\
\hline $\begin{array}{l}\text { Âge au début du } \\
\text { placement étudié }\end{array}$ & $F=2,91$ & 0,091 & 15,6 & 15,6 & 0,915 & $F=0,510$ & 0,477 & $F=0,128$ & 0,721 \\
\hline $\begin{array}{l}\text { \% de temps passé } \\
\text { hors du CR }\end{array}$ & - & - & 38,6 & 23,4 & 0,001 & $F=31,02$ & 0,000 & $F=6,75$ & 0,011 \\
\hline $\begin{array}{l}\text { Milieu de vie après } \\
\text { le placement } \\
\text { étudié (jours) } \\
\text { Famille } \\
\text { Ressource CJQ } \\
\end{array}$ & - & - & - & - & - & $\begin{array}{l}11,8 \\
67,0\end{array}$ & 0,001 & $\begin{array}{l}15,7 \\
56,6\end{array}$ & 0,000 \\
\hline $\begin{array}{l}\text { Nombre de jours } \\
\text { placés au suivi } \\
3 \text { mois }\end{array}$ & - & - & - & - & - & - & - & $F=55,84$ & 0,000 \\
\hline
\end{tabular}

Résultats des analyses de régression sur les mesures d'effets

Les tableaux suivants présentent les résultats des analyses de régression multivariée sur les quatre mesures d'effets retenues pour le programme "Transfert des acquis". Dans le modèle explicatif, nous avons retenu seulement les variables qui présentent une contribution significative (valeur $p<0,05$ ). 
Tableau 4

Résultats de l'analyse de régression multivariée sur le pourcentage de temps passé à l'extérieur du centre de réadaptation

\begin{tabular}{|l|c|c|c|c|c|}
\cline { 2 - 6 } \multicolumn{1}{c|}{} & \multicolumn{5}{c|}{$\begin{array}{c}\text { Analyse de variance : Valeur F : 9,92 } \\
\text { Valeur } \mathbf{p}: \mathbf{0 , 0 0 0} \\
\mathbf{R}^{2} \text { ajusté : 0,133 }\end{array}$} \\
\hline $\begin{array}{l}\text { Variables / } \\
\text { Statistiques }\end{array}$ & $\begin{array}{c}\text { Coefficient } \\
\text { de } \\
\text { régression b }\end{array}$ & Écart-type & $\boldsymbol{t}$ & $\boldsymbol{P}$ & $\begin{array}{c}\mathbf{R}^{2} \\
\text { partiel }\end{array}$ \\
\hline $\begin{array}{l}\text { Groupe } \\
\text { (0= témoin } \\
1=\text { TDA) } \\
\begin{array}{l}\text { Âge au début } \\
\text { du placement } \\
\text { Constante }\end{array}\end{array}$ & 14,59 & 3,69 & 3,94 & 0,000 & 0,12 \\
\hline
\end{tabular}

Les résultats sur le pourcentage de temps passé à l'extérieur du centre de réadaptation (tableau 4) montrent que la variable groupe augmente de $14,59 \%(b=14,59)$ le pourcentage de temps passé à l'extérieur du centre de réadaptation, alors que l'âge diminue ce temps de $4,54 \%(b=-4,54)$, pour chaque unité d'âge qui augmente. Ce dernier résultat laisse supposer que, plus le jeune est âgé au moment de sa participation, plus le temps passé en dehors du centre de réadaptation diminue. II semble donc y avoir un effet attribuable au fait de participer au groupe TDA et au fait d'être plus jeune lors du placement. Ce résultat confirme l'objectif du "Transfert des acquis » d'augmenter le temps de séjour en dehors du centre de réadaptation en cours de placement. II faut cependant noter que la valeur du $\mathrm{R}^{2}$ est faible $(0,133)$, ce qui veut dire qu'en dépit d'un modèle explicatif statistiquement significatif ce dernier ne peut réussir à prédire qu'une partie limitée des résultats obtenus.

Pour la variable d'effet qui concerne le milieu de vie après le placement nous avons utilisé la technique de régression logistique, laquelle est requise lorsque la variable dépendante implique seulement deux catégories, dans ce cas-ci : famille ou ressource Centre jeunesse de Québec. Nous avons également utilisé comme variable indépendante le pourcentage de temps passé en 
dehors du centre de réadaptation. Cette variable est présente dans les deux groupes TDA et TÉMOIN; c'est pourquoi il faut tenter de saisir son rôle spécifique indépendamment de la variable groupe. L'interprétation des coefficients de régression $b$ dans le cas d'une analyse de régression logistique diffère quelque peu de celle dans le cas d'une régression linéaire. Dans la régression logistique, le coefficient de régression $b$ de chaque variable contribue au calcul de la probabilité de se retrouver dans la catégorie codée 1 de la variable dépendante, dans ce cas-ci, donc, la probabilité de se retrouver en ressource du Centre jeunesse de Québec (ressource CJQ) après le placement étudié (Menard, 1995). Le tableau 5 illustre les résultats obtenus à l'analyse de la régression logistique en termes de probabilités. Comme on peut le constater, la probabilité de se retrouver en centre jeunesse diminue de manière importante à mesure qu'augmente le pourcentage de temps passé à l'extérieur du centre de réadaptation. Ainsi, lorsque que l'on double le pourcentage de temps passé en dehors du centre de réadaptation, on peut diminuer de 6 à 7 fois la probabilité de se retrouver en ressource du Centre jeunesse. Cette probabilité passe de 0,084 à 0,013 pour le groupe TDA et de 0,022 à 0,003 pour le groupe TÉMOIN. On peut également constater qu'à pourcentage égal de temps passé en dehors du centre de réadaptation les probabilités sont plus grandes pour le groupe TDA de se retrouver en ressource du centre jeunesse, et ce, dans un rapport de 4 à 1 . II semble donc que, si les probabilités varient selon la variable groupe, le pourcentage de temps passé en dehors du centre de réadaptation influence également de manière importante ces mêmes probabilités. Ce résultat ne va pas tout à fait dans le sens des prédictions que l'on aurait pu faire concernant la participation au groupe TDA. Par contre, la contribution significative du temps passé à l'extérieur du centre renforce l'hypothèse du "Transfert des acquis » à l'égard du renforcement du temps passé en milieu familial comme stratégie efficace d'intervention. 


\section{Tableau 5 \\ Probabilité de se retrouver en ressource du \\ Centre jeunesse de Québec selon le groupe et différents pourcentages de temps passé en dehors du centre de réadaptation}

\begin{tabular}{|c|c|c|}
\hline $\begin{array}{c}\text { Pourcentage de temps passé } \\
\text { en dehors du centre de } \\
\text { réadaptation }\end{array}$ & $\begin{array}{c}\text { Groupe } \\
\text { « Transfert } \\
\text { des acquis } ~\end{array}$ & $\begin{array}{c}\text { Groupe } \\
\text { TÉmolN }\end{array}$ \\
\hline $30 \%$ & 0,084 & 0,022 \\
\hline $45 \%$ & 0,039 & 0,010 \\
\hline $65 \%$ & 0,013 & 0,003 \\
\hline
\end{tabular}

Nous avons effectué le même type d'analyses (non présentées) pour les deux autres variables, soit le nombre de jours placés 3 mois après la fin du placement et le nombre de jours placés entre le troisième et le sixième mois après le placement étudié. Les résultats suggèrent que la variable groupe n'a pas de lien significatif avec ces deux variables. La variable qui semble le plus déterminer le nombre de jours placés après 3 mois est le milieu de vie après le placement. Si le milieu de vie immédiatement après le placement étudié est le centre jeunesse, le nombre de jours placés en ressource du centre jeunesse au suivi 3 mois est de 31,6 jours sur une période de 93 jours. De plus, pour chaque augmentation d'une unité de pourcentage de temps passé en dehors du centre de réadaptation, on peut diminuer de 0,594 jour le séjour en centre de réadaptation après 3 mois. Le modèle construit explique $47,9 \%$ de la variance de la variable dépendante.

En ce qui concerne la dernière variable, le nombre de jours placés entre le troisième et le sixième mois, le modèle explicatif compte pour $41,8 \%$ de la variance de la variable dépendante. La variable pourcentage de temps passé en dehors du centre de réadaptation ne présentant pas de relation significative avec le suivi entre le troisième et le sixième mois, elle a été retirée du modèle final. Par contre, pour chaque journée placée au suivi 3 mois, 0,67 jour s'ajoute aux journées placées entre le troisième et le sixième mois. Ainsi, plus un jeune est replacé dans les 3 mois qui suivent 
le placement, plus il a de chances de continuer à être placé entre 3 et 6 mois après le placement.

\section{EN CONCLUSION SUR LES ANALYSES...}

Les résultats des quatre analyses de régression ne permettent pas de conclure de façon formelle à un effet soutenu de la variable groupe en lien avec les mesures d'effets retenus. Le groupe TDA est une variable explicative importante dans le cas du pourcentage de temps passé à l'extérieur du Centre jeunesse de Québec, ce qui va dans le sens des objectifs des promoteurs d'augmenter le temps passé à l'extérieur du centre de réadaptation au profit de séjours en famille. Cette variable perd cependant de sa force à mesure que l'on s'éloigne dans le temps. Ainsi, le milieu de vie par la suite ne dépend strictement pas de l'appartenance au "Transfert des acquis ». On observe même la tendance inverse, à savoir que les probabilités de se retrouver au Centre jeunesse de Québec sont plus élevées lorsqu'on est du groupe TDA. II est possible que ces résultats soient attribuables à des problèmes de recrutement de la clientèle TDA. Certains jeunes ont commencé le programme, mais ont dû soit en cours de route ou à la fin de leur participation continuer leur séjour en centre de réadaptation parce qu'ils ne répondaient pas bien au programme. Les résultats confirment par ailleurs l'importance du temps passé à l'extérieur comme variable explicative pour le milieu de vie après le placement et au suivi 3 mois.

En dépit du fait que la variable groupe ait des difficultés à conserver un rôle certain sur les mesures d'effets, le modèle explicatif du " Transfert des acquis » n'est pas pour autant invalidé. Le fait que le temps passé à l'extérieur soit en lien avec les mesures d'effets renforce l'hypothèse que le "Transfert des acquis " favorise des séjours à la maison sur une base assidue, augmentant les chances de retourner dans la famille et donc de diminuer le nombre de jours placés au suivi 3 mois. Nous pensons donc que, même si les résultats ne se présentent pas de manière idéale, les relations entre les variables nous apparaissent suffisamment étoffées pour valider l'hypothèse principale du programme «Transfert des acquis" 


\section{Estimations sur la gestion des places et les coûts évités en réadaptation}

À partir des résultats présentés ci-dessus, nous avons tenté d'estimer quels pouvaient être leurs impacts sur la gestion des places de réadaptation au Centre jeunesse de Québec. Cette estimation ne couvre évidemment pas toute la complexité de la réalité des services de réadaptation, ni du processus décisionnel qui procède au placement. Cependant, les bénéfices en termes de jours passés à l'extérieur du centre de réadaptation ainsi que les jours en moins placés après 3 et 6 mois suggèrent des pistes de réflexion.

Le tableau 6 présente les résultats de gains en termes de libération de lits (nuitées) selon différents pourcentages de temps passé en dehors du centre de réadaptation. Les hypothèses émises ici concernent la libération des lits lorsque le jeune couche à l'extérieur du centre de réadaptation. Évidemment, plus les pourcentages de séjours à la maison sont élevés et plus il y aura de places disponibles. En les convertissant sur une base annuelle, on arrive à un volume de nuitées disponibles pour accommoder d'autres jeunes soit en dépannage ou en placement court terme. Le nombre de nuitées estimé est calculé sur une base de séjour moyen de 164 jours. C'est une estimation à la hausse de ce qui pourrait être disponible pour une unité. En effet, malgré les séjours en famille possibles, les éducateurs doivent soutenir ces jeunes sur une base quasi quotidienne si l'on désire maintenir l'implication des familles. Si l'on transformait le nombre de nuitées supplémentaires estimé ici en places pour des jeunes, il est évident que cela aurait des répercussions sur la qualité du travail effectué auprès des autres jeunes et que cela diminuerait les gains potentiels. En fait, il faudrait plutôt se référer au nombre de nuitées potentielles disponibles et imaginer quelle pourrait être la meilleure utilisation de celles-ci sur une base annuelle. L'utilisation optimale de ces gains suppose une gestion serrée des places disponibles à tout moment dans les unités et également la mise en œuvre d'un programme de type "Transfert des acquis » qui renforce et soutient les séjours à la maison. 
Tableau 6

Estimations des gains de libération de lits (nuitées) selon diverses hypothèses de temps passé à l'extérieur du centre de réadaptation lors du placement

\begin{tabular}{|c|c|c|c|}
\hline $\begin{array}{l}\text { Pourcentage } \\
\text { de temps } \\
\text { passé à } \\
\text { l'extérieur } \\
\text { du C.R. }\end{array}$ & $\begin{array}{c}\text { Gain moyen } \\
\text { par } \\
\text { séjour par } \\
\text { jeune } \\
(\% \times 164)\end{array}$ & $\begin{array}{c}\text { Gain } \\
\text { annualisé } \\
\text { par jeune } \\
(\% \times 164) \div \\
164 \times 365\end{array}$ & $\begin{array}{c}\text { Gain annuel pour } \\
12 \text { jeunes "Transfert } \\
\text { des acquis » } \\
([\% \times 164] \div 164 \times \\
365) \times 12 \text { jeunes }\end{array}$ \\
\hline $28 \%$ & 46 nuitées & 102 nuitées & 1224 nuitées \\
\hline $42 \%$ & 68 nuitées & 151 nuitées & 1812 nuitées \\
\hline $75 \%$ & 123 nuitées & 273 nuitées & 3276 nuitées \\
\hline
\end{tabular}

Le même type d'exercice a été effectué pour estimer les coûts supplémentaires évités 3 mois après la fin du placement (tableau 7). À partir du paramètre " pourcentage de temps passé à l'extérieur du centre de réadaptation » $(b=-0,594)$ identifié dans les analyses de régression, nous avons calculé le nombre de jours de placement épargnés selon diverses hypothèses. Ainsi, toutes autres variables étant égales par ailleurs, si des jeunes passent $28 \%$ de leur temps de placement à l'extérieur du centre de réadaptation, on calcule $28 \times 0,594=16$ jours de placement évités par jeune au suivi 3 mois. Nous avons également supposé qu'avec un séjour moyen de 164 jours par jeune 24 jeunes pouvaient transiter dans une unité au cours d'une année. Ainsi, l'estimation pour 24 jeunes est près d'une projection sur une base annuelle des coûts évités. Le tarif journalier du Centre jeunesse $(150,00 \$)$ a été appliqué aux résultats pour estimer les coûts évités. Le tableau 7 donne les résultats de ces compilations. L'effet net du «Transfert des acquis » est la différence entre les coûts estimés à $42 \%$ et ceux à $28 \%$, qui représente le groupe TÉMOIN, soit (90000\$ - $567000 \$=43300 \$)$. Le programme "Transfert des acquis " permettrait donc de " gagner » environ 43300 \$ sur une base annuelle pour une unité en augmentant le temps passé à l'extérieur du centre de réadaptation, soit de $28 \%$ à $42 \%$. 
Tableau 7

Estimation des coûts évités 3 mois après la fin du placement pour 24 jeunes, selon le pourcentage de temps passé en dehors du centre de réadaptation (séjour moyen de 164 jours)

\begin{tabular}{|c|c|c|}
\hline $\begin{array}{c}\text { Pourcentage } \\
\text { de temps } \\
\text { passé à } \\
\text { l'extérieur } \\
\text { du C.R. }\end{array}$ & $\begin{array}{c}\text { Gain de jours placés } \\
\mathbf{3} \text { mois après la fin du } \\
\text { placement par jeune } \\
(\% \times \mathbf{0 , 5 9 4 )}\end{array}$ & $\begin{array}{c}\text { Coûts évités } 3 \text { mois } \\
\text { après la fin du placement } \\
\text { pour 24 jeunes } \\
\text { (base annuelle) }\end{array}$ \\
\hline $28 \%$ & $\begin{array}{c}\text { (Gain de jours à 3 mois } \\
\times 24 \text { jeunes } \times \text { taux journalier } \\
\text { du CJQ) }\end{array}$ \\
$42 \%$ & 16 jours & $57600 \$$ \\
$75 \%$ & 25 jours & $90000 \$$ \\
44 jours & $158400 \$$ \\
\hline
\end{tabular}

Note : Le taux journalier du Centre jeunesse de Québec est estimé à 150 \$.

En dépit des limites méthodologiques imposées par le devis utilisé et par le statut expérimental du programme, nous avons pu mettre en valeur la contribution spécifique du programme "Transfert des acquis " à l'augmentation du temps de séjour dans leur famille des jeunes placés. De plus, ce temps placé augmente significativement les probabilités de se retrouver en famille après le placement ainsi que le nombre de jours placés 3 mois après la fin du placement. Cette dernière variable influence elle-même le nombre de jours placés entre 3 et 6 mois. Ces résultats permettent de penser que le programme "Transfert » des acquis, au-delà des bénéfices cliniques, contribue également à augmenter la disponibilité des lits en internat et donc à éviter certains coûts attribuables à l'engorgement périodique auquel doit faire face le centre de réadaptation.

\section{RÉSULTATS DES ENTREVUES SUR LES EFFETS DE L'IMPLICATION PARENTALE AVEC LES PARTICIPANTS AU PROGRAMME}

L'analyse faite du contenu des entrevues réalisées avec les différents informateurs clés (parents, jeunes, éducateurs, intervenants et gestionnaires) fait ressortir que l'implication plus grande des parents constitue un élément crucial des changements amenés 
par le programme "Transfert des acquis ». Les différents acteurs sont d'accord pour dire qu'il s'agit d'un avantage significatif pour tout le monde.

Le fait de partager les torts dans la situation familiale problématique permet au jeune de se sentir libéré du fardeau de la responsabilité des problèmes. La participation de ses parents au processus de réadaptation permet un partage de la charge des efforts à effectuer afin d'améliorer leur situation dans le but d'une réinsertion familiale plus rapide. Le jeune a ainsi une vision moins punitive du placement étant donné que lui et ses parents travaillent ensemble dans un but commun de réinsertion familiale.

Par ailleurs, les parents sont heureux de se sentir impliqués, d'être consultés et de retrouver ainsi leur rôle de parents. L'implication parentale dans le processus de réadaptation permet ainsi aux parents de se sentir soutenus par les intervenants dans l'exercice de leurs rôles parentaux.

L'implication parentale plus grande est aussi un avantage pour les intervenants dont la tâche s'est modifiée en s'orientant davantage vers les " vrais problèmes ». En effet, les intervenants ont l'impression que leur travail est devenu plus valorisant du fait qu'ils se sont penchés sur les difficultés auprès de tous les membres du système-famille. La présence plus fréquente des parents dans les unités des centres de réadaptation a aussi permis aux éducateurs de voir leur tâche réduite.

La volonté d'impliquer les parents suppose l'exercice d'un tutorat élargi. En effet, les éducateurs continuent de s'occuper du jeune, mais ils travaillent maintenant aussi avec les parents dans une vision plus globale du problème à régler. Les activités d'apprentissage contribuent à donner aux parents un rôle dans le processus de réadaptation et de faire en sorte que les intervenants soient un soutien plutôt qu'un "substitut parental ". Aussi, avec le "Transfert des acquis ", les parents du jeune placé ont appris à s'impliquer dans la gestion des conflits, des tensions et ils ont dû faire des efforts afin que les problèmes disparaissent, même si leur jeune était placé. Le placement n'est plus synonyme de congé ou de répit pour les parents, car ceux-ci participent tout autant que leur jeune dans la résolution de leurs problèmes. 
L'implication parentale plus grande vient en somme répondre à l'objectif de départ du "Transfert des acquis " qui était de faire tout ce qui est possible afin de planifier la réinsertion familiale dès le début du placement.

Certaines situations constituent cependant des obstacles au bon déroulement du programme. C'est le cas notamment pour les problèmes de toxicomanie. En effet, certains jeunes et leurs parents considèrent que le "Transfert des acquis » n'a pas été efficace dans leur cas parce que les jeunes n'étaient pas motivés à s'impliquer dans le programme en raison de leurs problèmes de toxicomanie. Certains parents pensent également au fait que leur jeune n'ait pas pu retourner vivre avec eux à la fin du programme, mais ils ne blâment pas le programme quant à sa pertinence. Ils disent plutôt que leur fils n'a peut-être pas été mis à la bonne place au bon moment et suggèrent qu'une évaluation plus précise des besoins et de la motivation du jeune à retourner vivre avec ses parents soit faite non seulement en début de programme, mais à d'autres moments tout au long du placement.

Pour ce qui est de l'orientation « milieu » qui constituait un autre objectif de départ du programme "Transfert des acquis", les résultats sont moins convaincants. En effet, le travail plus systémique auprès du réseau scolaire, social et communautaire du jeune n'a pas été aussi soutenu que l'objectif de départ le voulait. Des parents et des jeunes le mentionnent et trouvent important que le jeune puisse demeurer le plus possible dans ses activités régulières de loisirs, avec ses amis, sans briser ses liens avec son milieu d'origine; il semble cependant que trop peu de jeunes conservent effectivement le lien avec leur milieu. Cet aspect du programme "Transfert des acquis " a été très peu développé et peu du temps de travail des intervenants y a été consacré. De plus, la question des activités actualisantes à cet égard n'a pas été clairement comprise et, par conséquent, l'application des principes a été difficile. Les intervenants reconnaissent qu'il reste encore beaucoup de travail à faire dans cette branche du programme. Les mêmes constatations s'appliquent pour le milieu scolaire. Les intervenants se sont très peu préoccupés de cet aspect. Plusieurs jeunes du TDA ont quand même pu bénéficier de l'encadrement scolaire offert au centre de réadaptation. 
La formule des activités d'apprentissage en groupe a été appréciée par les parents, de même que par plusieurs intervenants. Elle l'a cependant été un peu moins par les jeunes. Les intervenants trouvent valorisant de voir que les parents "embarquent " dans ces activités et semblent même en redemander. Presque tous les parents trouvent ces rencontres très enrichissantes : ils y trouvent leur profit; elles leur font du bien en tant que parents, car ils voient qu'ils ne sont pas les seuls parents à éprouver des difficultés avec leur jeune. Ils en jugent le contenu généralement très pertinent. Quant aux jeunes, les activités d'apprentissage en groupe ne les enthousiasment pas beaucoup. Comme ils ont généralement de la difficulté à comprendre les liens entre les thèmes de discussion et leurs situations personnelles, ils restent difficilement impliqués. De plus, la majorité des jeunes ne savent pas trop quoi répondre à la question de savoir ce qu'ont donné les activités d'apprentissage ou ce qu'elles leur ont permis d'acquérir en matière de connaissances ou d'habiletés grâce à ces rencontres. II semble donc, selon tous les acteurs consultés, que les activités d'apprentissage rejoignent plus les parents que les jeunes, et ce, tant sur le plan de la satisfaction face aux rencontres que des résultats donnés par les rencontres. Ainsi, l'objectif de départ des activités d'apprentissage, qui était de donner des outils aux jeunes et aux parents pour augmenter leurs compétences en communication, est atteint du côté des parents, alors qu'il ne semble pas l'avoir été du côté des jeunes.

Les différents acteurs du programme sont d'accord pour dire que la gestion des situations de crise s'est faite beaucoup plus efficacement, en faisant tous les efforts possibles pour que la crise se règle dans la famille sans avoir recours au retrait en isolement dans l'institution comme c'était le cas auparavant. Les efforts en ce sens des intervenants constitue une nouveauté dans leur intervention et plusieurs jeunes de même que plusieurs parents ont grandement apprécié cette nouvelle façon d'intervenir.

D'un point de vue organisationnel, l'implantation de "Transfert des acquis » répond aux préoccupations actuelles des gouvernements d'améliorer l'efficacité et l'efficience des programmes de réadaptation. Le programme permet une gestion plus souple des places disponibles en centre de réadaptation. Comme le jeune passe plus de temps dans son milieu naturel, les éducateurs 
peuvent suivre plus de jeunes qu'il y a de places dans les unités. Par exemple, dans une unité, les éducateurs ont pu suivre quinze jeunes, alors que seulement douze places sont inscrites officiellement.

En modifiant le profil de placement, le «Transfert des acquis » a permis de changer le rôle du centre de réadaptation. Ce dernier n'est plus considéré comme un milieu de vie où le jeune s'installe, mais il devient plutôt un filet de sécurité utilisé en cas de besoin seulement. Ainsi, au début du placement, le jeune ne sortait que les fins de semaine, mais progressivement il passe une proportion de temps de plus en plus grande à la maison et donc à l'extérieur du centre. Certains parents se sont même vu réduire leur contribution financière reliée au placement en fonction du temps réellement passé en centre de réadaptation. II y a donc eu un changement de mentalité quant au rôle du centre de réadaptation qui va dans le sens de l'objectif de réduire au maximum l'utilisation du centre de réadaptation.

Mais l'implantation d'un tel programme ne se fait pas sans quelques difficultés organisationnelles, comme l'ont soulevé les intervenants. En effet, ces derniers ont trouvé difficile d'implanter un programme faisant appel à une mentalité très différente de celle qui avait cours en centre de réadaptation, et ce, à l'intérieur des structures de fonctionnement existantes. Cette mise en place a nécessité de la formation, mais la plupart des intervenants auraient souhaité en recevoir davantage avant la mise en marche du programme afin de bien comprendre et de bien rendre opérationnels certains concepts, en particulier celui des activités actualisantes. Tous les éducateurs rencontrés ont déclaré que de s'occuper des parents autant que du jeune est, du point de vue de l'intervention clinique, un travail différent qui aurait demandé plus de supervision, d'encadrement et de soutien de la part, par exemple, des conseillers cliniques, des travailleurs sociaux ou des chefs d'équipe.

\section{CONCLUSION}

Dans le difficile contexte de transformation du réseau de la santé et des services sociaux, il n'est pas aisé de trouver le dynamisme nécessaire à l'expérimentation de nouveaux modes de distribution 
des services aux jeunes et au changement des mentalités qui les accompagnent. La subvention du Fonds de soutien à l'innovation du ministère de la Santé et des Services sociaux a permis de dégager une fenêtre d'opportunités dans le quotidien de l'intervention des praticiens en centre de réadaptation. Cette bouffée d'oxygène a aidé le personnel et l'organisation du Centre jeunesse de Québec à faire un pas en avant dans l'amélioration des services offerts aux jeunes et aux familles en difficulté dans la région de Québec.

Gilles MIREAULT

Institut universitaire sur les jeunes en difficulté Centre jeunesse de Québec

André BEAUDOIN Institut universitaire sur les jeunes en difficulté Centre jeunesse de Québec Professeur, Université Laval

Gaétan PAQUET Institut universitaire sur les jeunes en difficulté Centre jeunesse de Québec

Élise CHAMPAGNE Institut universitaire sur les jeunes en difficulté Centre jeunesse de Québec 


\section{Références bibliographiques}

Allerhand, M.E., R. Weber et M. Haug (1986). Adaptation and adaptability: the Bellefaire follow-up study, New York, Child Welfare League of America.

BeAudoin, A. et R. Aucoin (1996). Première phase de l'implantation du projet "Transfert des acquis ". Rapport d'évaluation, Québec, Institut universitaire sur les jeunes en difficulté. Centre jeunesse de Québec, $111 \mathrm{p}$.

Berkowitz, B.P. et A.M. Graziano (1972). "Training Parents as Therapists: A Review », Behavior research and therapy, vol. 10, p. 200-209.

BLYTHE, B.J., M.J. JoRdANA et S.A. Kelly (1991). « Family Preservation with Substance Abusing Families: Help that Works », Children and Family Services Quarterly, vol. 14, n³, p. 12-13.

CONSEIL DES AFFAIRES SOCIALES (1990). De la protection des enfants au soutien des parents. Québec, Éditeur officiel du Québec.

FANSHEL, D. (1975). «Parental Visiting of Children in Foster Care : A Key to Discharge ? " Social Service Review, vol. 49, p. 493-514.

Johnson, C.A. et C. KATZ (1973). " Using Parents as Change Agents for Their Children: A Review », Journal of Child Psychology and Psychiatry, vol. 14, p. 181-200.

KRONA, D.A. (1980). "Parents as Treatment Partners in Residential Care », Child Welfare, 59, p. 91-96.

KWANTES, C. (1992). "Re-thinking Residential Child Care: Working Systemic Within the Constraints of Residential Treatment », Journal of Child and Youth Care, vol. 7, $\mathrm{n}^{\circ}$ 3, p. 33-44.

LANTZ, J.E. (1987). "Family Intervention During Residential Treatment », Residential Treatment for Children and Youth, vol. 4, $\mathrm{n}^{\circ}$ 4, p. 39-52.

LEBLANC, M. (1995). " Y a-t-il trop d'adolescents placés en internat aux Centres jeunesse de Montréal ? ", Revue canadienne de psychoéducation, vol. 24, n 2 , p. 93-120.

MÉNARD, S. (1995). Applied Logistic Regression Analysis, Series: Quantitative applications in the Social Sciences, Thousand Oaks, Sage, $97 \mathrm{p}$. 
Mireault, G., A. Beaudoin, G. Paquet et É. Champagne (1998). Transfert des acquis : rapport final d'évaluation, Québec, Institut universitaire sur les jeunes en difficulté, Centre jeunesse de Québec, 149 p.

PAQUET, G. (1995). "Transfert des acquis »: conceptualisation du modèle, Québec, Centre jeunesse de Québec, $35 \mathrm{p}$.

PAUZÉ, R., D. BÉCHARD et J. TOUPIN (1993). «Facteurs de risques associés au placement d'enfants en milieu substitut: revue de la littérature récente dans ce domaine », Apprentissage et socialisation, vol. $16, n^{\circ} 3$, p. 203-213.

RoBeRge, P. (1995). Le placement des mineurs au Québec: quelques éléments d'information, Québec, ministère de la Santé et des Services sociaux, vol. 9, $\mathrm{n}^{\circ}$ 4, p. 39-50.

Rowe, J., H. CAin, M. Hundleby et A. KeAne (1984). Long-term Foster Care, Londres, Basford Academic and Educational.

SIMARD, M., J. VACHON et M. MOISAN (1991). La réinsertion familiale de l'enfant placé : facteurs de succès et d'échec, Québec, Université Laval, Centre de recherche sur les services communautaires.

TARDIF, J. (1992). Pour un enseignement stratégique, l'apport de la psychologie cognitive, Montréal, Éditions Logiques.

TAYLOR, D.A. et S.W. AlPERT (1973). Continuity and Support Following Residential Treatment, New York, Child Welfare League of America.

WHITTAKER, J.K. (1981). " Family Involvement in Residential Treatment », dans A. Maluccio et P. A. Sinanoglu (dir.), The Challenge of Partnership: Working with Parents of Children in Residential Care, New York, Child Welfare League of America, Inc.

WhitTAKeR, J.K. et A.N. Maluccio (1986). Position Paper on Out-ofHome Care: Prepared for the Resolutions Committee, Biennial Meeting of Child Welfare League of America, Unpublished Manuscript. University of Washington, School of Social Work, Seattle, novembre.

WHITTAKER, J.K. et J. GARBARINo (1983). Social Supports Networks, New York, Aldine de Gruyter. 
